Cronfa - Swansea University Open Access Repository

This is an author produced version of a paper published in :

ACS Nano

Cronfa URL for this paper:

http://cronfa.swan.ac.uk/Record/cronfa21239

\title{
Paper:
}

Tang, L., Ji, R., Li, X., Bai, G., Liu, C., Hao, J., Lin, J., Jiang, H., Teng, K., Yang, Z. \& Lau, S. (2014). Deep Ultraviolet to Near-Infrared Emission and Photoresponse in Layered N-Doped Graphene Quantum Dots. ACS Nano, 8(6), 6312 6320.

http://dx.doi.org/10.1021/nn501796r

This article is brought to you by Swansea University. Any person downloading material is agreeing to abide by the terms of the repository licence. Authors are personally responsible for adhering to publisher restrictions or conditions. When uploading content they are required to comply with their publisher agreement and the SHERPA RoMEO database to judge whether or not it is copyright safe to add this version of the paper to this repository. http://www.swansea.ac.uk/iss/researchsupport/cronfa-support/ 


\section{Deep Ultraviolet to Near-Infrared Emission and Photoresponse in Layered N-Doped Graphene Quantum Dots}

Libin Tang, ${ }^{\dagger, t}$ Rongbin Ji, ${ }^{*}$ Xueming Li, ${ }^{\S}$ Gongxun Bai, ${ }^{\dagger}$ Chao Ping Liu, ${ }^{\dagger}$ Jianhua Hao $^{\dagger}$

Jingyu Lin, 'Hongxing Jiang, ${ }^{\prime}$ Kar Seng Teng, ${ }^{\perp}$ Zhibin Yang ${ }^{\dagger}$ and Shu Ping Lau ${ }^{\dagger *}$

† Department of Applied Physics, The Hong Kong Polytechnic University, Hong Kong SAR.

† Kunming Institute of Physics, Kunming, 650223, Yunnan Province, P.R. China.

$\S$ Solar Energy Research Institute, Yunnan Normal University, Kunming, 650092, P.R. China

॥Department of Electrical and Computer Engineering, Texas Tech University, Lubbock, Texas 79409, United States

$\perp$ Multidisciplinary Nanotechnology Center, College of Engineering, Swansea University, Singleton Park, Swansea SA2 8PP, United Kingdom

* To whom correspondence should be addressed. E-mail: apsplau@polyu.edu.hk. 


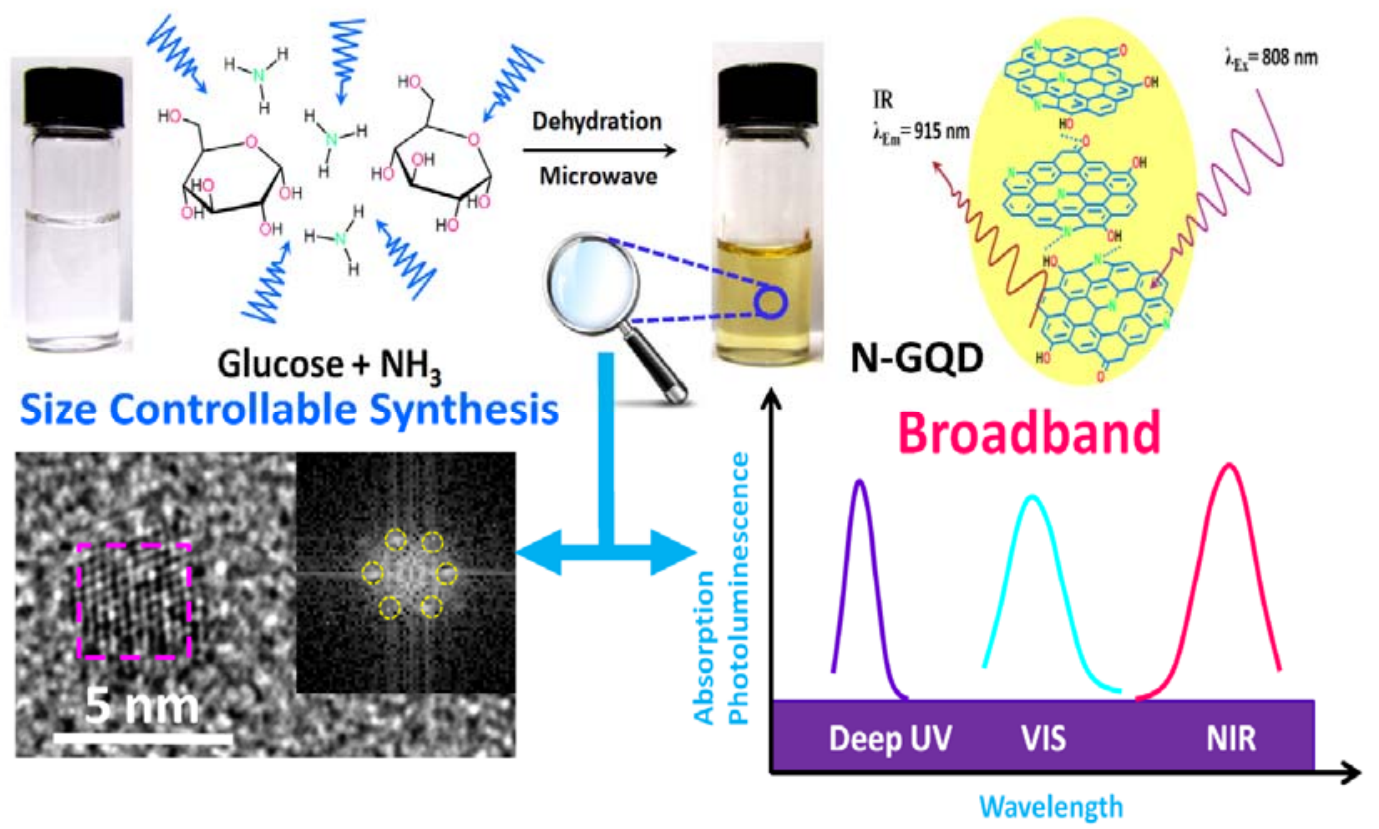

ABSTRACT: Material that can emit a broad spectral wavelengths covering deep ultraviolet (DUV), visible, and near infrared (NIR) is highly desirable. It can lead to important applications such as broadband modulators, photodetectors, solar cells, bioimaging, and fiber communications. However, there is currently no material that meets such desirable requirement. Here, we report the layered structure of nitrogen-doped graphene quantum dots (N-GQDs) which possess broadband emission ranging from $300 \mathrm{~nm}$ to over $1000 \mathrm{~nm}$. The broadband emission is attributed to the layered structure of the N-GQDs that contains a large conjugated system and provides extensive delocalized $\pi$ electrons. In addition, a broadband photodetector with responsivity as high as $325 \mathrm{~V} / \mathrm{W}$ is demonstrated by coating N-GQDs onto interdigital gold electrodes. The unusual negative photocurrent is observed which is attributed to the trapping sites induced by the self-passivated surface states in the N-GQDs. 
KEYWORDS: Graphene quantum dot, nitrogen doping, photoluminescence, broadband emission, photoresponse

Materials that exhibit broadband absorption and emission covering deep ultraviolet (DUV), visible and near infrared (NIR) spectral range are of scientific and technological importance due to its valuable applications in the fields of broadband photodetectors, solar cells, and bioimaging. Currently, different semiconductor materials are required to cover these broad spectral ranges. Narrow-gap inorganic semiconductors such as $\mathrm{PbS},{ }^{1} \mathrm{PbSe},{ }^{2} \mathrm{PbTe},{ }^{3} \mathrm{HgTe},{ }^{4}$ and $\mathrm{InGaAs}^{5}$ show infrared optical properties, but their toxicity and chemical instability limit their applications. While the wide-bandgap semiconductors such as $\mathrm{ZnO},{ }^{6} \mathrm{GaN},{ }^{7}$ and $\mathrm{AlN}^{8}$ based materials possess UV and DUV optical properties. Although the rare-earth element doped GaN materials exhibit NIR emission, ${ }^{9-11}$ tuning of these materials are difficult for UV applications. Therefore, it is highly desirable to develop a novel functional material that is capable of absorbing and emitting DUV, visible, and NIR spectral wavelengths.

Graphene $\mathrm{e}^{12-14}$ is a hexagonal honeycomb single layer $\mathrm{sp}^{2}$ bonded carbon material. It exhibits a broadband absorption ranging from 300 to $2500 \mathrm{~nm}^{14}$ which is beneficial to ultrafast broadband photodetectors. ${ }^{15-16}$ Due to its zero bandgap, graphene shows no optical emission and this limits its application in optoelectronic devices. However, graphene quantum dots (GQDs) have emerged as an attractive fluorescence material in visible and DUV spectral range, ${ }^{17}$ but NIR emission has yet to be reported. Nitrogen-doped graphene quantum dot (N-GQD) is a functional material that can be prepared by a number of methods, ${ }^{18-22}$ such as electrochemical, ${ }^{18}$ organic synthesis, ${ }^{19}$ hydrothermal, ${ }^{20-21}$ and self-catalysis. ${ }^{22}$ The electrocatalytic activity, ${ }^{18-19}$ tunable luminescence, ${ }^{20}$ and biocompatibility ${ }^{20}$ of N-GQDs have been demonstrated. Here we report a facile "one-pot" microwave-assisted method to prepare N-GQDs using glucose and aqueous 
ammonia as sources. Most interestingly, the N-GQDs exhibit broadband emission ranging from $300 \mathrm{~nm}$ to over $1000 \mathrm{~nm}$. The emission bandwidth of the N-GQDs is one of the widest among all the semiconductor materials and their quantum dot counterparts. The broadband emission is attributed to the layered structure of the N-GQDs which consists of a large conjugated system containing extensive delocalized $\pi$ electrons. Furthermore, the N-GQDs exhibited broadband photoresponse with unusual negative photocurrent when irradiated by various wavelengths ranging from 365 to $980 \mathrm{~nm}$. Due to the low-cost, chemical stability as well as the broadband emission and photoresponse properties, the N-GQDs should find important applications in broadband photodetectors, solar cells, bioimaging, and fiber communications.

\section{RESULTS AND DISCUSSION}

The monodispersed N-GQDs with various sizes were prepared by microwave assisted hydrothermal method (MAH) using glucose and aqueous ammonia as sources. The synthetic process is schematically shown in Figure 1a. Glucose provides carbon source. Ammonia plays two roles in the growth of the N-GQDs, firstly, it acts as catalyst for the intra-molecular and inter-molecular dehydration of glucose, and secondly it is for $\mathrm{N}$ doping.

The TEM image of the N-GQDs prepared at a growth time of 3 min is shown in Figure $1 \mathrm{~b}$. The prepared N-GQDs show monodispersed distribution. The N-GQDs highlighted by the red dashed circles reveal the uniformity in size. The size distribution obeys a Gaussian distribution; the most probable size is $1.9 \mathrm{~nm}$ with a full-width-at-half-maximum (FWHM) of $0.25 \mathrm{~nm}$. The size of the N-GQDs increases with increasing heating time, for example, as the growth time increases from 1 to $9 \mathrm{~min}$, the diameter of the N-GQDs increases from $1.7 \mathrm{~nm}$ to $5.8 \mathrm{~nm}$. The heating time dependence accords with an exponential growth process as shown in Figure 1c. The crystalline 
structure of the N-GQDs with sizes of $1.7,1.9,3.0,4.0$ and $5.8 \mathrm{~nm}$ prepared at the heating time of $1,3,5,7$ and 9 min respectively are shown in Figure 1d. In order to investigate the morphology and the height of the N-GQDs, atomic force microscopy (AFM) characterization was performed, as shown in Figure 1e for N-GQDs grown for 3 min. The dots A, B, C, and D indicated by the blue arrows are randomly chosen N-GQDs, their heights range from 1.6 to 1.7 $\mathrm{nm}$ (inset of Figure 1e), which are very close to the size of the N-GQDs $(1.88 \mathrm{~nm}$ ) as characterized using TEM.

Figure $2 \mathrm{a}$ is the high-resolution transmission electron microscopy (HRTEM) image of N-GQD prepared by 5 min heating. The lattice fringes of the N-GQD can be clearly observed. The fast Fourier transform (FFT) of the selected area (pink square) is shown in the inset of Figure 2a, revealing the hexagonal crystalline structure. The N-GQD also reveals a layered structure (Figure $2 \mathrm{~b}$ ) with an interlayer spacing of $\sim 0.38 \mathrm{~nm}$ (Figure $2 \mathrm{c}$ ) which is slightly larger than that of bulk graphite $(0.335 \mathrm{~nm})^{23-24}$ due to the presence of the functional groups that enlarge the basal plane spacing of the N-GQDs.

The detailed TEM structural characterization of the N-GQDs can be found in Supporting Information (SI, Figures S1-S5) which reveal the high-crystal quality of the N-GQDs. The electron energy loss spectroscopy (EELS) ${ }^{17,25-29}$ and low-loss EELS of the N-GQDs were studied and the detailed discussion can be found in SI (Figure S6 and Figure S7, respectively).

X-ray photoelectron spectroscopy (XPS) $)^{30-31}$ were performed on the samples to investigate the chemical bonding of the N-GQDs. Figure S8a of SI shows the N 1s XPS spectra as a function of size. Three types of N-related bonding can be identified, namely pyridinic $\mathrm{N}(\sim 399.2 \mathrm{eV})$, pyrrolic $\mathrm{N}(\sim 400.2 \mathrm{eV})$, and graphitic $\mathrm{N}(\sim 401.6 \mathrm{eV})$. These peaks are also observed in NGQDs prepared by self-catalysis method..$^{22}$ The C 1s XPS spectra are shown in Figure S8b of SI. 
The spectra can be deconvoluted into five peaks centered at around $284.5 \mathrm{eV}(\mathrm{C}=\mathrm{C}), 285.8 \mathrm{eV}$ $(\mathrm{C}-\mathrm{C}, \mathrm{C}-\mathrm{H}, \mathrm{C}=\mathrm{N}), 286.6 \mathrm{eV}(\mathrm{C}-\mathrm{OH}), 287.2 \mathrm{eV}(\mathrm{C}-\mathrm{O}-\mathrm{C}, \mathrm{C}-\mathrm{N})$ and $288.6 \mathrm{eV}(\mathrm{C}=\mathrm{O})$, respectively, revealing different types of bonding to $C$. The atomic ratio N/C of the N-GQDs $(\Phi=3.0 \mathrm{~nm})$ is determined to be $8.3 / 100$, indicating $\mathrm{C}$ is the dominant element. The $\mathrm{N} / \mathrm{C}$ atomic ratio $(8.3 \%)$ is much higher than that of various $\mathrm{N}$-doped graphene-based materials $(0.3-5.6 \%) .{ }^{21,32-33}$ The NGQDs were further characterized by x-ray diffraction (XRD), Fourier-transform infrared (FTIR) spectroscopy and Raman spectroscopy as discussed in SI (Figure S9).

The UV-Vis-NIR absorption spectra of the N-GQDs are shown in Figure 3. There are three UV absorption peaks located at $214.5 \mathrm{~nm}, 268.0 \mathrm{~nm}$, and $310.5 \mathrm{~nm}$. The UV absorption peaks are related to the electron transitions from $\pi$ (or $n$ ) to $\pi^{*}$ of $\mathrm{C}=\mathrm{C}, \mathrm{C}=\mathrm{N}$, and $\mathrm{C}=\mathrm{O}$. It is worth noting that the N-GQDs with diameter larger than $1.7 \mathrm{~nm}$ exhibit a broad visible absorption. The visible absorption band between 400 and $700 \mathrm{~nm}$ is highlighted in blue as shown in Figure 3 . Thus, the origin of visible absorption of the N-GQDs could be related to the partial conjugated $\pi$ electrons in the layered N-GQDs. Importantly, a broad NIR absorption band peaked at $\sim 812 \mathrm{~nm}$ become noticeable as the size of the N-GQDs reaches $3.0 \mathrm{~nm}$. The NIR absorption could be attributed to the larger conjugated system containing extensive delocalized $\pi$ electrons in the layered N-GQDs. The UV-Vis-NIR absorption spectra suggest that the layered structure of the N-GQDs with relatively larger size $(\sim 3.0 \mathrm{~nm})$ can lead to visible and NIR absorption which is due to an increase in conjugated $\pi$ electrons in the structure.

The photoluminescence (PL) quantum yields (QYs) of the N-GQDs with various diameters were determined to be between 6.8\% and 11.3\% using an absolute QY measurement method. The excitation wavelength $\left(\lambda_{E}\right)$ of $275 \mathrm{~nm}$ was used and the QY spectra were measured between 400 and $700 \mathrm{~nm}$. The PL excitation (PLE) spectra of the N-GQDs are shown in Figure S10 of SI. 
Three bands are located at $239 \mathrm{~nm}, 269 \mathrm{~nm}$, and $333 \mathrm{~nm}$. These peaks correspond to the electron transitions from $\pi$ (or $n$ ) to $\pi^{*}$ of $\mathrm{C}=\mathrm{C}, \mathrm{C}=\mathrm{N}$, and $\mathrm{C}=\mathrm{O}$. Clearly, these values are close to those of the UV absorptions, showing the electron transitions for double bonds in the N-GQDs occur in the UV range. Importantly, the N-GQDs exhibit broadband PL peaked at $302 \mathrm{~nm}, 542 \mathrm{~nm}$ and $915 \mathrm{~nm}$ when excited by wavelengths of $197 \mathrm{~nm}, 475 \mathrm{~nm}$ and $808 \mathrm{~nm}$ respectively, as shown in Figure 4. This is the first graphene-based material which exhibits broadband PL covering the entire DUV-visible-NIR spectral range.

Figure 5a shows the PL spectra of the N-GQDs at various sizes using $197 \mathrm{~nm}$ laser as an excitation source measured at $300 \mathrm{~K}$. The size of the N-GQDs does not apparently influence the DUV peak centered at $\sim 302 \mathrm{~nm}$ (PL spectra measured at $10 \mathrm{~K}$ are shown in Figure S11 of SI). The size independent DUV peak may be due to the localized $\pi$ electrons of the $\mathrm{C}$ based double bonds (mainly $\mathrm{C}=\mathrm{C}$ ) in the N-GQDs. In addition to the DUV emission, we also observed a visible light emission ( $>400 \mathrm{~nm}$ ), which is resulted from the radiative recombination after interband transition that converts DUV absorption to visible light emission. Figure $5 \mathrm{~b}$ shows the size-dependent visible PL emission of the N-GQDs excited by $375 \mathrm{~nm}$ laser. For the N-GQDs with diameter of $1.7 \mathrm{~nm}$, the emission peak is centered at $450 \mathrm{~nm}$. The emission peak red shifts as the size increases. The wavelength reaches $582 \mathrm{~nm}$ when the size equals to $5.8 \mathrm{~nm}$. Figure $5 \mathrm{c}$ shows the NIR PL spectra of the N-GQDs excited by a laser with wavelength of $808 \mathrm{~nm}$. If the size of the N-GQDs is too small $(\sim 1.7 \mathrm{~nm})$, no obvious NIR emission can be observed, probably due to the small conjugated system which contains fewer delocalized $\pi$ electrons. As the size of the N-GQDs increased to $\geq 3.0 \mathrm{~nm}$, there are two NIR emissions peaked at $\sim 862 \mathrm{~nm}$ and $\sim 917$ $\mathrm{nm}$. These emissions are attributed to free and bound excitons. The bound excitons were bound to the surface states (defects), requiring additional coulomb energies which make the bound 
excitons energy lower (i.e. $917 \mathrm{~nm})$ than that of the free excitons $(862 \mathrm{~nm})$. Importantly, the NIR emission peaks can be further tuned to a longer wavelength when the N-GQDs was excited by a light source with longer wavelength, as shown in Figure S12 of SI. The NIR emission peaks at $\sim 1017$ and $\sim 1060 \mathrm{~nm}$ can be observed when the N-GQDs were excited by a laser of 980nm. Similar to the typical GQDs, the N-GQDs also exhibited excitation wavelength $\left(\lambda_{\mathrm{Ex}}\right)$ dependent PL as shown in Figure S13a of SI. Both the PL intensity and emission peak position changes with $\lambda_{\mathrm{Ex}}$ (Figure S13b of SI). ${ }^{26}$

Based on the information of the structural, absorption and PL properties, the mechanism of the broadband emission covering DUV, visible and NIR range can be explained as follows. Figure 6 shows the schematic diagrams of the broadband emission mechanism of the N-GQDs excited by various wavelengths. As for the DUV emission, the absorption of DUV photons by the localized $\pi$ electron in double bonds (mainly $\mathrm{C}=\mathrm{C}$ ) produces electron-hole pair (exciton) after electron transition $\left(v_{3} \rightarrow c_{3}\right)$. The exciton may emit DUV light $\left(c_{3} \rightarrow v_{3}\right)$ through radiative recombination after vibration relaxation (Figure 6d). The excited electron may also undergo interband transition from a higher conduction band to a lower conduction band (e.g., $\left.c_{3} \rightarrow c_{2}\right)$, subsequently emit visible light $\left(c_{2} \rightarrow v_{2}\right)$ by radiative recombination. This could be the reason why the DUV absorption resulted in both DUV and visible emission as shown in Figure 5a. As far as visible emission is concerned, the partial conjugated $\pi$ electrons in the layered structure of the N-GQD (Figure 6 b) play a major role. The visible light absorption $\left(v_{2} \rightarrow c_{2}\right)$ of the partial conjugated $\pi$ electrons may result in excitons which can emit visible light $\left(c_{2} \rightarrow v_{2}\right)$ (Figure 6e) after the vibration relaxation that emit the photons with wavelength longer than the absorbed wavelengths. A longer excitation wavelength is needed for a larger conjugated $\pi$ electron system in order to satisfy the most probable absorption, resulting in excitation wavelength $\left(\lambda_{\mathrm{Ex}}\right)$ dependent emission. 
In the case of NIR emission, the conjugated $\pi$ electrons in the layered structure of the N-GQDs facilitate the NIR absorption (Figure 6c), the vibration relaxation should also occur since the wavelength of the emitted NIR light (e.g., $915 \mathrm{~nm}$ ) (Figure 6f) is longer than the wavelength of the absorbed NIR photons $(808 \mathrm{~nm})$. Apparently, only those N-GQDs with size reached $3.0 \mathrm{~nm}$ can absorb and emit NIR light. The layered structure assists the process due to the extensive conjugated $\pi$ electron system. It is reported that $\pi$ electron $^{34}$ and large macromolecular domains ${ }^{35}$ are also related to the NIR PL mechanism observed in graphene oxide (GO).

The time-resolved PL decay measurements were performed on the N-GQDs. A tripleexponential equation ${ }^{17,36-37}$ is fitted well to the experimental data, the PL lifetime contains a fast component $\left(\tau_{1}: 0.61-1.38 \mathrm{~ns}\right)$ and two slow components $\left(\tau_{2}: 2.18-4.13 \mathrm{~ns}\right.$ and $\left.\tau_{3}>10 \mathrm{~ns}\right)$. The size-dependent PL decay curves at a fixed excitation wavelength $\lambda_{\mathrm{Ex}}(375 \mathrm{~nm})$ and emission wavelength $\lambda_{\mathrm{Em}}(570 \mathrm{~nm})$ are shown in Figure S14 of SI. It is found that the larger the N-GQDs the faster the decay time are. The average lifetimes and other fitting parameters for different $\mathrm{N}$ GQD sizes are listed in Table S1 of SI. The average lifetime strongly depends on the size of the N-GQDs. In order to reveal the size-dependent lifetime of the N-GQDs, the lifetime versus $\lambda_{\mathrm{Em}}$ for various sizes is plotted and shown in Figure S15a. It is evident that, a larger size N-GQD results in a shorter lifetime for a fixed $\lambda_{\mathrm{Em}}$. It can also be observed that the peak of the lifetime (maximum lifetime) increases with increasing N-GQD size. The above mentioned properties should correlate to the presence of $\mathrm{N}$ in the quantum dots. It is worth mentioning that under the same conditions $\left(\lambda_{\mathrm{Ex}}=375 \mathrm{~nm}, \lambda_{\mathrm{Em}}=470 \mathrm{~nm}\right)$, the average lifetimes of various N-GQD sizes are smaller than that of nitrogen-free GQDs. ${ }^{26}$ It suggests that the presence of $\mathrm{N}$ may effectively accelerate the process of emitting the photon after absorption, which is resulted from the "bridgeeffect" of $\mathrm{N}^{22}$ Combined with the structural information disclosed by XPS, the diversity of $\mathrm{N}$ 
configuration (pyridinic, pyrrolic and graphitic $\mathrm{N}$ ) is beneficial to the formation of conjugated $\pi$ electron system which may be responsible for the broadband emission in the N-GQDs.

In order to obtain an in depth understanding on the relationship between the PL lifetime and the size of the N-GQDs, a plot of lifetime versus diameter of the N-GQDs is shown in Figure S16a (SI). Evidently, an exponential decay curve is found. As mentioned above, the lifetime of the N-GQDs exhibits a "peak-like" dependence on $\lambda_{\text {Em }}$ which is similar to the dependence of PL emission intensity on wavelength. Both of the curves are similar in shape, but different in value (Figure S16b of SI). Under the same $\lambda_{\mathrm{Ex}}$ of $375 \mathrm{~nm}$, the PL emission peaked at $476 \mathrm{~nm}(\Phi=3.0$ $\mathrm{nm}$ ), the lifetime curve, however, peaked at $582 \mathrm{~nm}$, resulting in a red shift of $106 \mathrm{~nm}$. It can also be seen that the FWHM value of the lifetime curve $($ FWHM $=230 \mathrm{~nm})$ is wider than that of emission curve (FWHM $=154 \mathrm{~nm})$. The above differences in peak position and FWHM might be related to the doping and unique structure of the N-GQDs.

The N-GQDs also exhibit broad color tunability. Figure S15b shows the photographs of the NGQDs with different sizes under ambient (top) and $365 \mathrm{~nm}$ UV light (bottom) illumination. To illustrate the emission properties of the N-GQDs, DI water is used as a control sample. Clearly, the larger the size of the N-GQDs, the darker is the color of the solution. The N-GQDs can emit a variety of colors under UV light illumination, including blue, green-blue, white, green-yellow and orange. The chromaticity coordinates of the N-GQDs at different sizes were determined using a laser of $375 \mathrm{~nm}$ as the excitation source. As shown in Figure S15c, the letters A, B, C, D, and E denote the size of the N-GQDs of $1.7 \mathrm{~nm}, 1.9 \mathrm{~nm}, 3.0 \mathrm{~nm}, 4.0 \mathrm{~nm}$ and $5.8 \mathrm{~nm}$ respectively. Interestingly, a variety of emission colors can be obtained as the size of the N-GQDs increases from $1.7 \mathrm{~nm}$ to $5.8 \mathrm{~nm}$, for examples, blue $(A, 1.7 \mathrm{~nm})$, green-blue $(\mathrm{B}, 1.9 \mathrm{~nm})$, white $(\mathrm{C}, 3.0$ $\mathrm{nm})$, green-yellow $(\mathrm{D}, 4.0 \mathrm{~nm})$, and orange $(\mathrm{E}, 5.8 \mathrm{~nm})$. The corresponding chromaticity 
coordinates are listed in the inset of Figure S15c. The emission colors cover three primitive colors, therefore in principle it is possible to emit a variety of colors by mixing different sizes of N-GQDs. This should find application in light converters. ${ }^{17}$

The observed broadband emission properties in the N-GQDs have prompted us to study the photoresponse of the N-GQDs under various irradiation wavelengths. Figure 7 illustrates the fabrication processes of the N-GQDs based photodetectors using interdigital gold electrodes. The N-GQDs solution was drop-coated onto the interdigital electrodes, followed by heating at $90{ }^{\circ} \mathrm{C}$ for $15 \mathrm{~min}$ to stiffen the N-GQDs films on a hotplate in the air. The schematic diagram of the NGQDs based photodetector is shown in Figure 8a.

Different from conventional photodetectors, the N-GQDs exhibits negative photocurrent when irradiated to light as shown in Figure S17 of SI. When the light is turned on, the current is decreased rather than increased. When the light is off, the current is restored to its original level. In order to show the positive photoresponse of the N-GQDs, we measured the photovoltage of the detectors by applying a bias current to the detector. Figure $8 \mathrm{~b}$ shows the photovoltage response of the detector when irradiated with UV (365 nm, light emitting diode), visible (405 nm, laser), and NIR (808 $\mathrm{nm}$ and $980 \mathrm{~nm}$, laser) light sources. The N-GQDs photodetector can response to UV to NIR. The response time is about $50 \mathrm{~s}$ for $980 \mathrm{~nm}$ and reduced to about $10 \mathrm{~s}$ for $365 \mathrm{~nm}$. The performance parameters of the N-GQDs photodetector are listed in Table S2 of SI. The induced photovoltage $\left(V_{\text {photo }}\right)$ is the voltage difference between the peak $\left(V_{\text {peak }}\right)$ and valley ( $\left.V_{\text {valley }}\right)$ when light is turned on and off respectively. The on-off ratio is defined as $V_{\text {peak }} / V_{\text {valley. }}$ The voltage responsivity $\left(R_{v}\right)$ is calculated as $V_{\text {photo }} / P_{\text {light }}$, where $P_{\text {light }}$ is the power of the light source. ${ }^{38}$ The on-off ratios of $808 \mathrm{~nm}$ and $980 \mathrm{~nm}$ are 6.49 and 4.29 respectively, the values are much larger than 1 . The responsivities for various light sources are $4.79 \mathrm{~V} / \mathrm{W}(980 \mathrm{~nm}), 10.91$ 
V/W $(808 \mathrm{~nm}), 325.00 \mathrm{~V} / \mathrm{W}(405 \mathrm{~nm})$ and $1.14 \mathrm{~V} / \mathrm{W}(365 \mathrm{~nm})$. The responsivity values are larger than that of carbon nanotube based broadband photodetector $(0.9-1.8 \mathrm{~V} / \mathrm{W})$ with similar spectral range. ${ }^{38}$ The effect of bias current on the photovoltage response of the N-GQDs detector is shown in Figure $8 \mathrm{c}$. The responsivity increases linearly from 10.91 to $14.08 \mathrm{~V} / \mathrm{W}$ as bias current increases from 1 to $3 \mathrm{nA}$ (Figure S18 and Table S2 of SI). It should be noted that the performance of the photodetector has not been optimized yet. Based on the emission results, the photodetector may also respond to wavelengths below $365 \mathrm{~nm}$ and over $1000 \mathrm{~nm}$.

The observed photoresponse from the N-GQDs based detector can be explained as follows. The absorption of photon (UV-Vis-NIR) may generate excited electron-hole pairs (excitons) which cannot freely move due to the presence of passivated surface layer (functional groups) on the surface of the N-GQDs as revealed by XPS and FTIR. The bound electron-hole pairs may trap charge carriers when the N-GQDs are exposed to light, forming photoinduced charge traps (Figure S19 of SI). Under a bias current, due to the presence of photoinduced traps in the NGQDs, the charge carriers may be trapped drastically as they passed through the N-GQDs (Figure S20 of SI), in order to maintain a constant current, the trapped charges will be compensated by increasing voltage, resulting in increasing photovoltage.

\section{CONCLUSIONS}

The broadband emission from the N-GQDs covering $300 \mathrm{~nm}$ to over $1000 \mathrm{~nm}$ was demonstrated. The broadband optical properties of the N-GQDs are attributed to the layered structure of the N-GQDs which create a large conjugated system containing extensive delocalized $\pi$ electrons. The DUV emission comes from the localized $\pi$ electron in double bonds (mainly $\mathrm{C}=\mathrm{C}$ ). The visible emission is caused by the partial conjugated $\pi$ electrons in the $\mathrm{N}$ - 
GQDs. As for NIR emission, the conjugated $\pi$ electrons in the layered structure of the N-GQDs facilitate the NIR absorption. Furthermore, the N-GQDs exhibited photoresponse from $365 \mathrm{~nm}$ to $980 \mathrm{~nm}$ with responsibility as high as $325 \mathrm{~V} / \mathrm{W}$ at $405 \mathrm{~nm}$. The unique broadband emission and photoresponse of the layered N-GQDs may lead to applications in a wide range of optoelectronic devices including photodetectors, solar cells and light emitting devices.

\section{METHODS}

N-GQDs Preparation. One gram of glucose was dissolved in $7.5 \mathrm{~mL}$ deionized (DI) water, followed by adding $0.5 \mathrm{~mL}$ aqueous ammonia. After mixing the solution, the solution was diluted 5 folds with DI water. Then $2.5 \mathrm{~mL}$ of the source solution was taken out into a glass bottle. The solution was heated in a microwave oven at $280 \mathrm{~W}$ for $1,3,5,7$ and 9 min for growing a series of N-GQDs with various diameters. The N-GQDs samples were dialyzed by molecular weight cut-off (MWCO) of 1000 against DI water for characterizations. The yield of the N-GQDs was determined to be around $\sim 78 \%$.

Characterizations.TEM, HRTEM, HAADF-STEM, EDS, EELS, and low-loss EELS were performed on JEOL, JEM-2100F at operating voltage of $200 \mathrm{kV}$. The morphology and height characterizations of the N-GQDs were performed by atomic force microscope (AFM) (Digital Instruments NanoScope IV) operating in the tapping mode at room temperature. The FT-IR spectra of the samples were obtained using the KBr pellet method by Nicolet Magna-IR 760 spectrometer with a resolution of $4 \mathrm{~cm}^{-1}$. Raman scattering spectra were obtained using Horiba JobinYvon HR800 spectrometer at room temperature with a laser of $488 \mathrm{~nm}$ wavelength. XPS experiment was performed using $\mathrm{Al} \mathrm{K} \alpha$ source, having an energy of $1486.6 \mathrm{eV}$ at room 
temperature with the VG ESCAlab MKII. X-ray diffraction (XRD) was carried out with a Rigaku SmartLab X-ray diffractometer $(\mathrm{Cu} \mathrm{K} \alpha$ radiation $\lambda=1.54056 \AA)$ operating at $45 \mathrm{kV}$ and $200 \mathrm{~mA}$. The UV-Vis spectra were recorded at room temperature on a Shimadzu UV-2550 UVVis spectrophotometer. The PL characterizations including excitation spectra, time-resolved PL, as well as quantum yields of the N-GQDs were recorded using an FLS920P Edinburgh Analytical Instrument apparatus. For the excitation dependent emission spectra and PLE spectra, Xe lamp was used as an excitation source. The NIR emission spectra of the N-GQDs were measured using an $808 \mathrm{~nm}$ laser as excitation source. The DUV emission spectra of the N-GQDs films were investigated by employing a specially designed time-resolved DUV laser spectroscopy system consisting of a frequency tripled $(266 \mathrm{~nm})$ and quadrupled $(197 \mathrm{~nm}) \mathrm{Ti}-$ sapphire laser $(100 f s)$ with a $76 \mathrm{MHz}$ repetition rate. A streak camera with a time resolution of 2 ps was used for recording time-resolved PL. The absolute quantum yield measurements were carried out using an integrating sphere (Edinburgh instruments, $150 \mathrm{~mm}$ in diameter coated with barium sulphate). The N-GQDs samples were diluted with DI water to avoid reabsorption; the samples were placed in the cuvettes inside the integrating sphere. The time-resolved PL spectra of the N-GQDs were recorded using $375 \mathrm{~nm}$ picosecond pulsed laser (Model EPL375, Edinburgh Instruments) as an excitation source. The photoresponse measurements were carried out using a computer-controlled Keithley 2400 source meter connected to a probe station.

Conflict of Interest: The authors declare no competing financial interest. 
Acknowledgement. This work was financially supported by the Research Grants Council of Hong Kong (Project No. PolyU 5013/09P), HK PolyU grants (Project Nos. G-YN10, 1-ZV8N and 1-ZE14), the Key Project of Applied Basic Research of Yunnan Province (Project No. 2012FA003), and the National Natural Science Foundation of China (Grant Nos. 11374250, 61106098 and 61066004). JL and HJ acknowledge the support of NSF (DMR-1206652).

Supporting Information Available: The detailed information of chemicals, fabrication of photodetectors, structural characterization of N-GQD by TEM, EELS, low-loss EELS, XRD, FTIR and Raman, optical characterization by PL, PLE, time-resolved PL, chromaticity coordinates and the mechanism for photoresponse. This material is available free of charge via the Internet at http://pubs.acs.org.

\section{REFERENCES}

(1) Mcdonald, S. A.; Konstantatos, G.; Zhang, S.; Cyr, P. W.; Klem, E. J. D.; Levina, L.; Sargent, E. H. Solution-Processed PbS Quantum Dot Infrared Photodetectors and Photovoltaics. Nat. Mater.2005, 4, 138-142.

(2) Talapin, D. V.; Murray, C. B. PbSeNanocrystal Solids for n- and p-Channel Thin Film FieldEffect Transistors. Science2005, 310, 86-89.

(3) Urban, J. J.; Talapin, D. V.; Shevchenko, E. V.; Kagan, C. R.; Murray, C. B. Synergism in Binary Nanocrystal Superlattices Leads to Enhanced p-Type Conductivity in Self-Assembled $\mathrm{PbTe} / \mathrm{Ag}_{2}$ Te Thin Films. Nat. Mater.2007, 6, 115-121. 
(4) Keuleyan, S.; Lhuillier, E.; Guyot-Sionnest, P. Synthesis of Colloidal HgTe Quantum Dots for Narrow Mid-IR Emission and Detection. J. Am. Chem. Soc.2011, 133, 16422-16424.

(5) Patel, R. B.; Bennett, A. J.; Farrer, I.; Nicoll, C. A.; Ritchie, D. A.; Shields, A. J. Two-Photon Interference of the Emission from Electrically Tunable Remote Quantum Dots. Nat. Photonics, 2010, 4, 632-635.

(6) Soci, C.; Zhang, A.; Xiang, B.; Dayeh, S. A.; Aplin, D. P. R.; Park, J.; Bao, X. Y.; Lo, Y. H.; Wang, D. ZnO Nanowire UV Photodetectors with High Internal Gain. NanoLett.2007, 7, 1003-1009.

(7) Li, D.; Sun, X.; Song, H.; Li, Z.; Chen, Y.; Jiang, H.; Miao, G. Realization of a HighPerformance GaN UV Detector by Nanoplasmonic Enhancement. Adv. Mater.2012, 24, 845849.

(8) Oto, T.; Banal, R. G.; Kataoka, K.; Funato, M.; Kawakami, Y. 100 mW Deep-Ultraviolet Emission from Aluminium-Nitride-Based Quantum Wells Pumped by an Electron Beam. Nat. Photonics 2010, 4, 767-771.

(9) Hömmerich, U; Nyein, E. E.; Lee, D.S.; Heikenfeld, J. Steckl, A.J.; Zavada, J.M. Photoluminescence studies of rare earth (Er, Eu, Tm) in situ doped GaN. Mater. Sci. Eng. 2003, B105, 91-96.

(10) Kim, J. H.; Holloway, P. H. Near-Infrared Electroluminescence at Room Temperature from Neodymium-Doped Gallium Nitride Thin Films. Appl. Phys. Lett. 2004, 85, 1689-1691. 
(11) Kim, J. H.; Holloway, P. H. Near-Infrared-Electroluminescent Light-Emitting Planar Optical Sources Based on Gallium Nitride Doped with Rare Earths. Adv. Mater. 2005, 17, 91-96.

(12) Novoselov, K. S.; Geim, A. K.; Morozov, S. V.; Jiang, D.; Zhang, Y.; Dubonos, S. V.; Grigorieva, I. V.; Firsov, A. A. Electric Field Effect in Atomically Thin Carbon Films. Science 2004, 306, 666-669.

(13) Li, X.; Cai, W.; An, J.; Kim, S.; Nah, J.; Yang, D.; Piner, R.; Velamakanni, A.; Jung, I.; Tutuc, E.; Banerjee, S. K.; Colombo, L.; Ruoff, R. S. Large-Area Synthesis of High-Quality and Uniform Graphene Films on Copper Foils. Science 2009, 324, 1312-1314.

(14) Bonaccorso, F.; Sun, Z.; Hasan, T.; Ferrari, A. C. Graphene Photonics and Optoelectronics. Nat. Photonics 2010, 4, 611-622.

(15) Xia, F.; Mueller, T.; Lin, Y.-M.; Valdes-Garcia, A.; Avouris, P. Ultrafast Graphene Photodetector. Nat. Nanotech. 2009, 4, 839-843.

(16) Urich, A.; Unterrainer, K.; Mueller, T. Intrinsic Response Time of Graphene Photodetectors. Nano Lett. 2011, 11, 2804-2808.

(17) Tang, L.; Ji, R.; Cao, X.; Lin, J.; Jiang, H.; Li, X.; Teng, K. S.; Luk, C. M.; Zeng, S.; Hao, J.; Lau, S. P. Deep Ultraviolet Photoluminescence of Water-Soluble Self-Passivated Graphene Quantum Dots. ACS Nano 2012, 6, 5102-5110.

(18) Li, Y.; Zhao, Y.; Cheng, H.; Hu, Y.; Shi, G.; Dai, L.; Qu, L. Nitrogen-Doped Graphene Quantum Dots with Oxygen-Rich Functional Groups. J. Am. Chem. Soc.2012, 134,15-18. 
(19) Li, Q.; Zhang, S.; Dai, L.; Li, L.-S. Nitrogen-Doped Colloidal Graphene Quantum Dots and Their Size-Dependent Electrocatalytic Activity for the Oxygen Reduction Reaction.J. Am. Chem. Soc. 2012, 134, 18932-18935.

(20) Hu, C.; Liu, Y.; Yang, Y.; Cui, J.; Huang, Z.; Wang, Y.; Yang, L.; Wang, H.; Xiao, Y.; Rong, J. One-Step Preparation of Nitrogen-Doped Graphene Quantum Dots from Oxidized Debris of Graphene Oxide. J. Mater. Chem. B 2013, 1, 39-42.

(21) Li, M.; Wu, W.; Ren, W.; Cheng, H.-M.; Tang, N.; Zhong, W.; Du, Y. Synthesis and Upconversion Luminescence of N-Doped Graphene Quantum Dots. Appl. Phys. Lett. 2012, 101, No. 103107.

(22) Tang, L.; Ji, R.; Li, X.; Teng, K. S.; Lau, S. P. Energy-Level Structure of Nitrogen-Doped Graphene Quantum Dots. J. Mater. Chem. C 2013, 1, 4908-4915.

(23) Oshima, C.; Nagashima, A. Ultra-Thin Epitaxial Films of Graphite and Hexagonal Boron Nitride on Solid Surfaces. J. Phys.: Condens. Matter.1997, 9, 1-20.

(24) Biedermann, L. B.; Bolen, M. L.; Capano, M. A.; Zemlyanov, D.; Reifenberger, R. G. Insights into Few-Layer Epitaxial Graphene Growth on 4H-SiC(000ī) Substrates from STM Studies. Phys. Rev. B2009, 79, No. 125411.

(25) Gass, M. H.; Bangert, U.; Bleloch, A. L.; Wang, P.; Nair, R. R.; Geim, A. K. Free-Standing Graphene at Atomic Resolution. Nat. Nanotech. 2008, 3, 676-681.

(26) Tang, L.; Ji, R.; Li, X.; Teng, K. S.; Lau, S. P. Size-Dependent Structural and Optical Characteristics of Glucose-Derived Graphene Quantum Dots. Part. Part. Syst. Charact. 2013, 30, 523-531. 
(27) Li, X.; Lau, S. P.; Tang, L.; Ji, R.; Yang, P. Multicolour Light Emission from ChlorineDoped Graphene Quantum Dots. J. Mater. Chem. C 2013, 1, 7308-7313.

(28) Ci, L.; Song, L.; Jin, C.; Jariwala, D.; Wu, D.; Li, Y.; Srivastava, A.; Wang, Z. F.; Storr, K.; Balicas, L.; Liu, F.; Ajayan, P. M. Atomic Layers of Hybridized Boron Nitride and Graphene Domains. Nat. Mater. 2010, 9, 430-435.

(29) Mkhoyan, K. A.; Contryman, A. W.; Silcox, J.; Stewart, D. A.; Eda, G. Mattevi, C.; Miller, S.; Chhowalla, M. Atomic and Electronic Structure of Graphene-Oxide. NanoLett. 2009, 9, 1058-1063.

(30) Kim, S.; Zhou, S.; Hu, Y.; Acik, M.; Chabal, Y. J.; Berger, C.; Heer, W.; Bongiorno, A.; Riedo, E. Room-Temperature Metastability of Multilayer Graphene Oxide Films. Nat. Mater.2012, 11, 544-549.

(31) Tang, L.; Li, X.; Ji, R.; Teng, K. S.; Tai, G.; Ye, J.; Wei, C.; Lau, S. P. Bottom-UP Synthesis of Large-Scale Graphene Oxide Nanosheets. J. Mater. Chem.2012, 22, 56765683.

(32) Zhang, C.; Fu, L.; Liu, N.; Liu, M.; Wang, Y.; Liu, Z. Synthesis of Nitrogen-Doped Graphene Using Embedded Carbon and Nitrogen Sources. Adv. Mater.2011, 23, 1020-1024.

(33) Panchakarla, L. S.; Subrahmanyam, K. S.; Saha, S. K.; Govindaraj, A.; Krishnamurthy, H. R.; Waghmare, U. V.; Rao, C. N. R. Synthesis, Structure, and Properties of Boron- and Nitrogen-Doped Graphene. Adv. Mater. 2009, 21, 4726-4730.

(34) Luo, Z.; Vora, P. M.; Mele, E. J.; Johnson A. T. C.; Kikkawa, J. M. Photoluminescence and Band Gap Modulation in Graphene Oxide. Appl. Phys. Lett.2009, 94, No. 111909. 
(35) Sun, X.; Liu, Z.; Welsher, K.; Robinson, J. T.; Goodwin, A.; Zaric, S.; Dai, H. NanoGraphene Oxide for Cellular Imaging and Drug Delivery. Nano Res.2008, 1, 203-212.

(36) Peng, J.; Gao, W.; Gupta, B. K.; Liu, Z.; Romero-Aburto, R.; Ge, L.; Song, L.; Alemany, L. B.; Zhan, X.; Gao, G.; Vithayathil, S. A.; Kaipparettu, B. A.; Marti, A. A.; Hayashi, T.; Zhu, J.-J.; Ajayan, P. M. Graphene Quantum Dots Derived from Carbon Fibers. NanoLett.2012, 12, 844-849.

(37) Bao, L.; Zhang, Z. L.; Tian, Z. Q.; Zhang, L.; Liu, C.; Lin, Y.; Qi, B. P.; Pang, D. W. Electrochemical Tuning of Luminescent Carbon Nanodots: From Preparation to Luminescence Mechanism. Adv. Mater.2011, 23, 5801-5806.

(38) St-Antoine, B. C.; Ménard, D.; Martel, R. Single-Walled Carbon Nanotube Thermopile for Broadband Light Detection. Nano Lett. 2011, 11, 609-613. 


\section{FIGURES AND CAPTIONS}
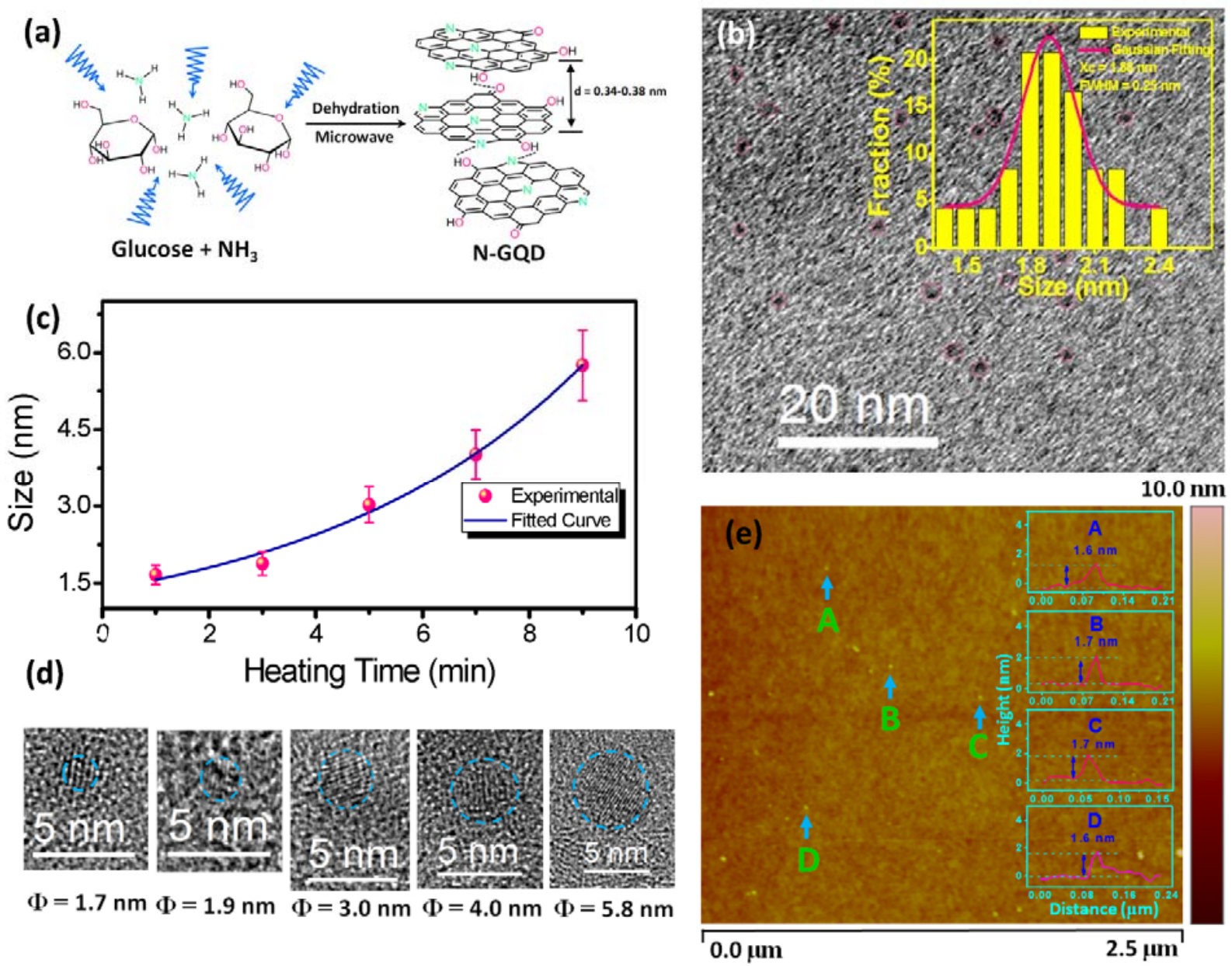

Figure 1. (a) The schematic representation of the synthesis of N-GQDs. (b) TEM image of the N-GQDs (3 min heating) assembled on $\mathrm{Cu}$ grid coated with ultrathin amorphous carbon film. Inset: the size distribution of the N-GQDs, the red line is the Gaussian fitting curve. (c) The size of the N-GQDs as a function of heating time. (d) The crystalline N-GQDs with sizes of $1.7 \mathrm{~nm}$, $1.9 \mathrm{~nm}, 3.0 \mathrm{~nm}, 4.0 \mathrm{~nm}$ and $5.8 \mathrm{~nm}$ were grown for $1 \mathrm{~min}, 3 \mathrm{~min}, 5 \mathrm{~min}, 7 \mathrm{~min}$ and $9 \mathrm{~min}$, respectively. (e) The morphology and height analyses of the N-GQDs as shown in AFM image, quantum dots A, B, C and D are randomly chosen N-GQDs. 


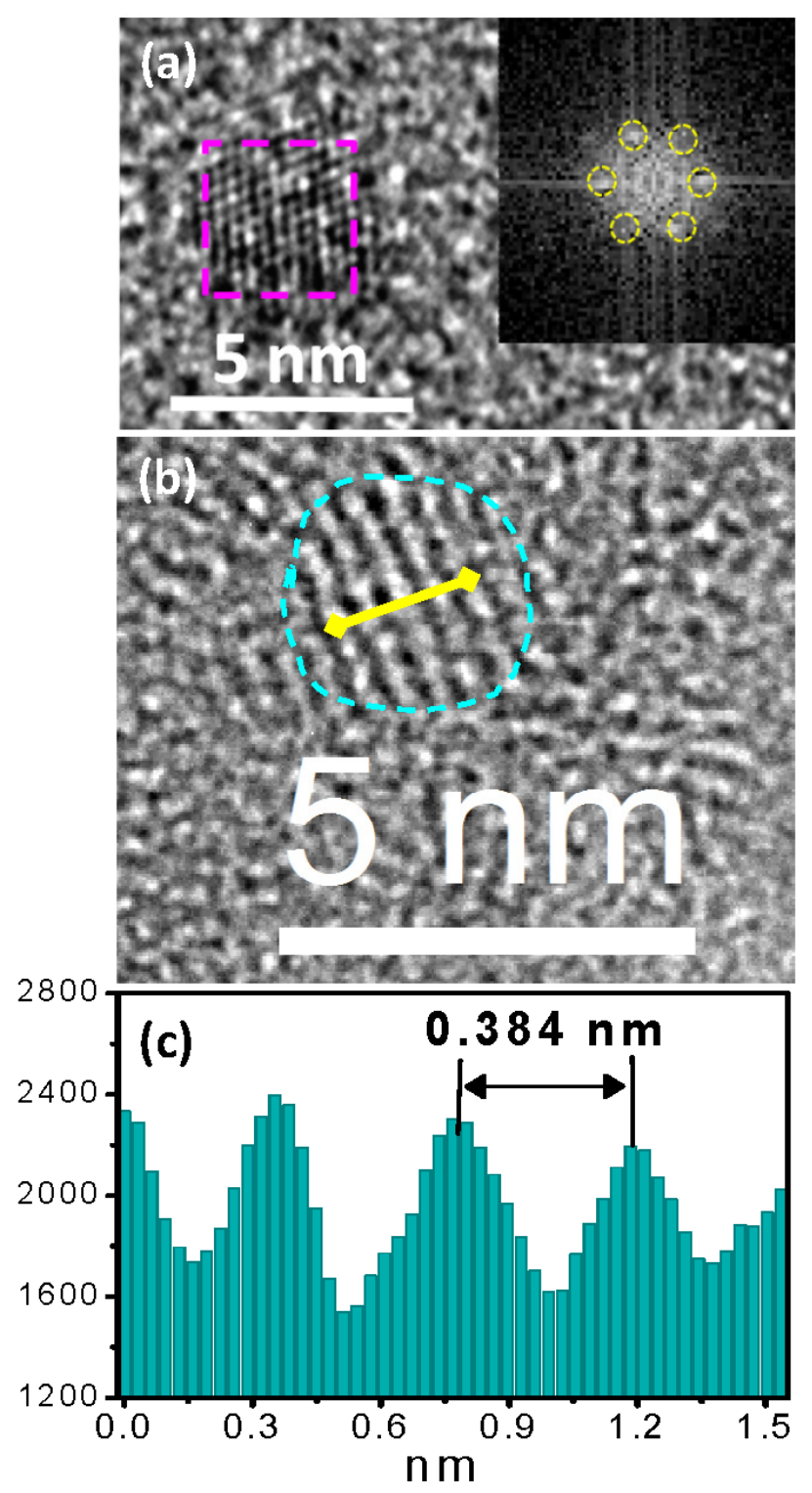

Figure 2. (a) HR-TEM image of a typical N-GQD. Inset: The fast Fourier transform (FFT) image of a selected area (pink square), the hexagonal honey comb structure is indicated. (b) The HRTEM image and the line profile (c) of the selected N-GQD. The layered structure with an interlayer spacing of $0.384 \mathrm{~nm}$ is observed. The sample was prepared by microwave heating for 5 min. 


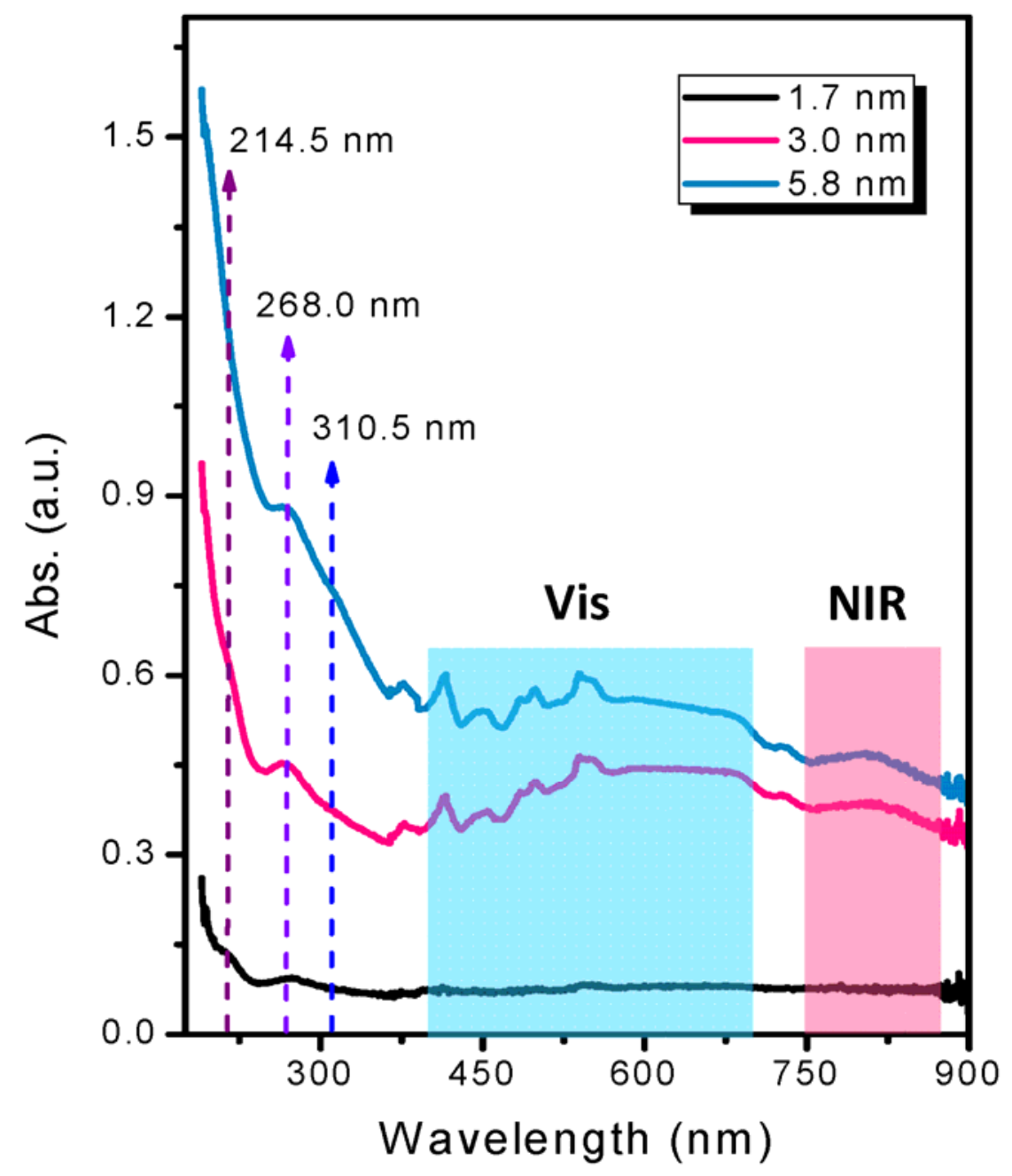

Figure 3.The UV-Vis-NIR absorption spectra of the N-GQDs at various sizes. 


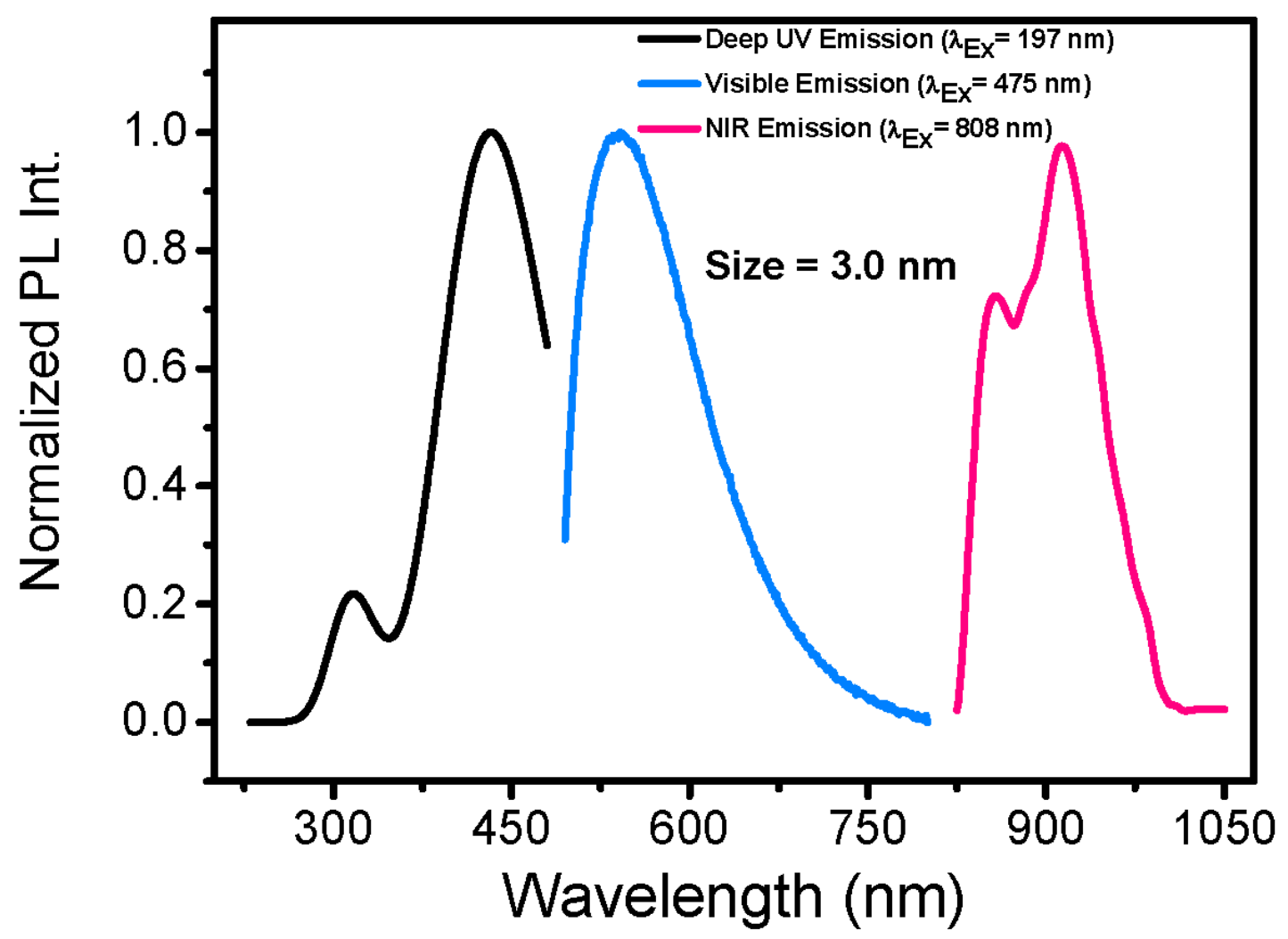

Figure 4. The normalized PL spectra of the N-GQDs $(\Phi=3.0 \mathrm{~nm})$ excited by various wavelengths. 

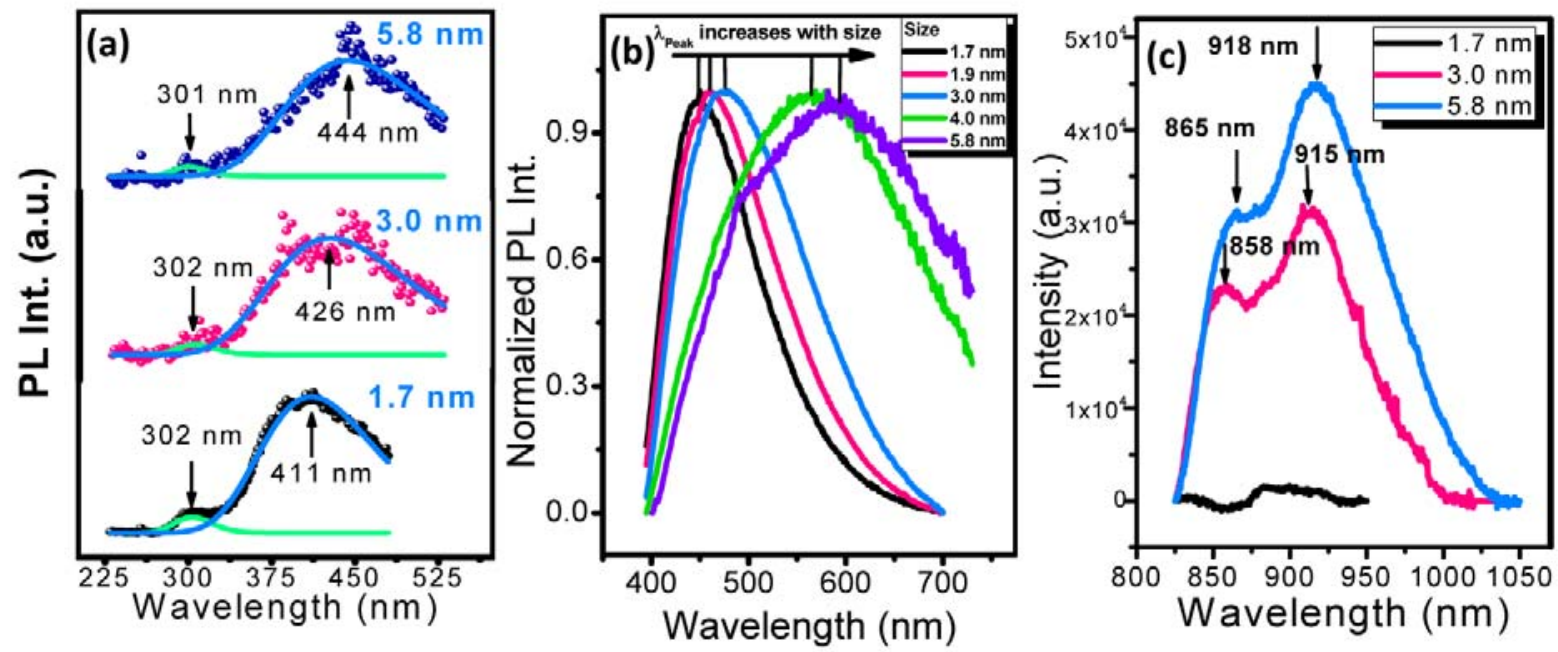

Figure 5. The PL spectra of the N-GQDs with different sizes. (a) Deep UV PL spectra of the NGQDs excited by $197 \mathrm{~nm}$ laser measured at $300 \mathrm{~K}$. (b) The size-dependent visible PL spectra of N-GQDs excited by $375 \mathrm{~nm}$ laser. (c) The NIR PL spectra of the N-GQDs excited by $808 \mathrm{~nm}$ laser. 


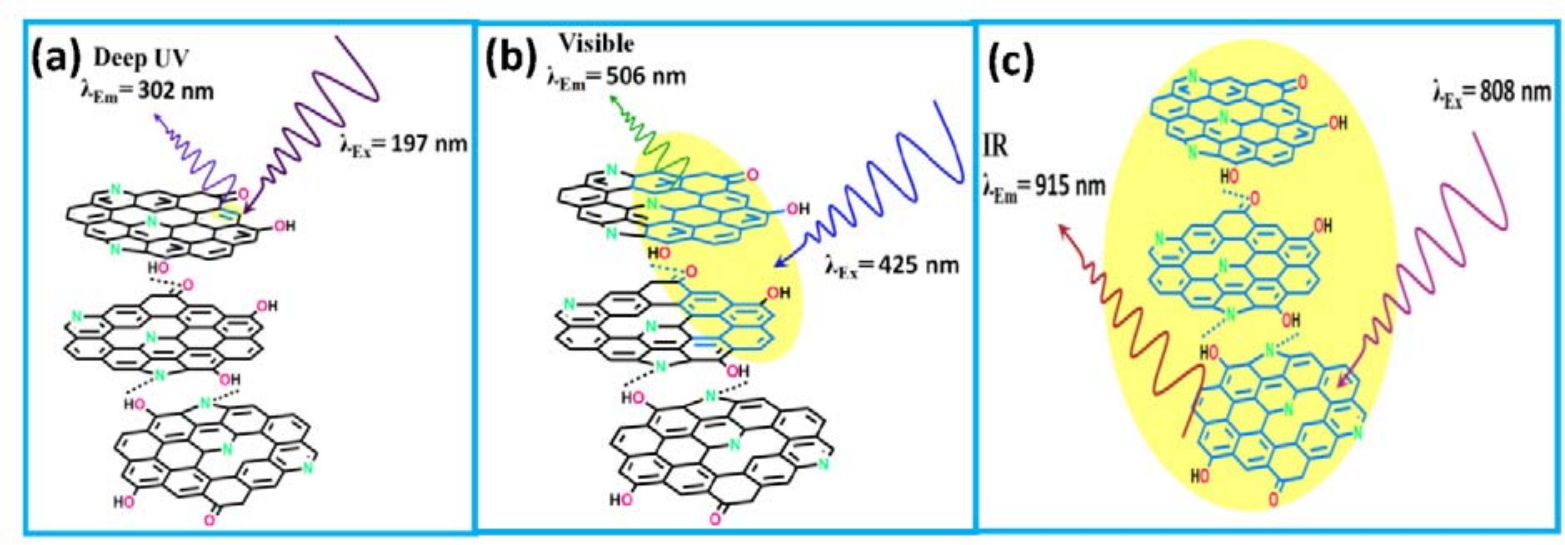

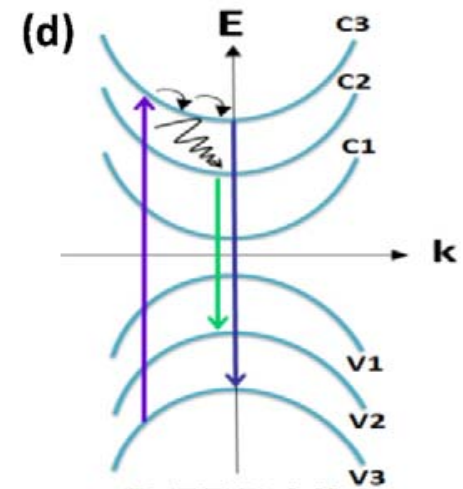

DEEP U $\mathbf{V}^{\mathbf{3}}$ (e)

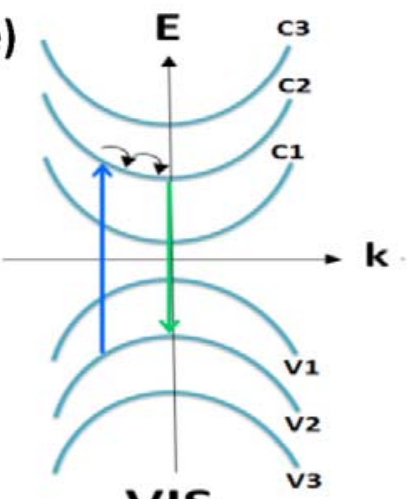

VIS (f)

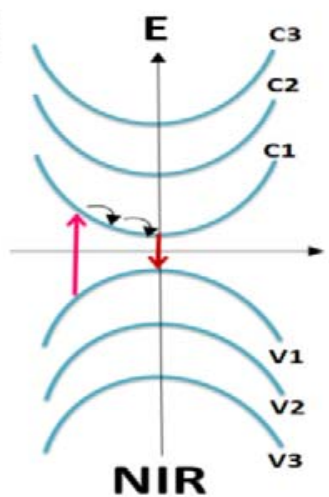

Figure 6. The mechanism for the broadband emission in the N-GQDs. (a) The DUV absorption by localized $\pi$ electron in carbon based double bonds (mainly $\mathrm{C}=\mathrm{C}$ ), the DUV PL emission and visible light PL emission occur after vibration relaxation and interband transition shown in (d). (b) The visible light absorption by partial conjugated $\pi$ electrons in the layered structure of the GQDs, the $\lambda_{\text {Ex }}$ dependent visible light PL emission occurs after vibration relaxation as shown in (e). (c) The NIR absorption by larger conjugated system containing more $\pi$ electrons in the layered structure of the N-GQDs, the NIR PL emission occurs after vibration relaxation as shown in (f). 

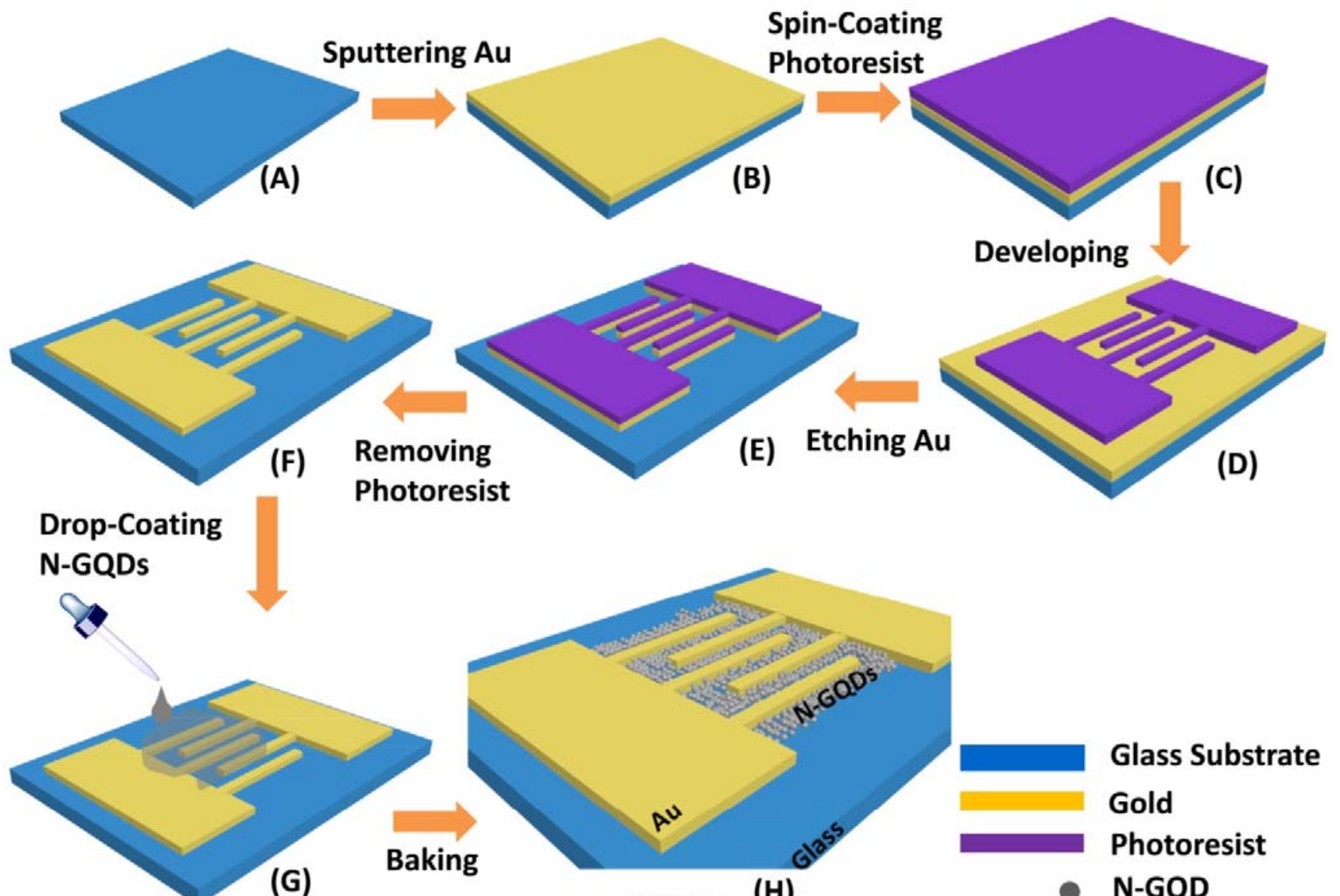

N-GQDs (H)

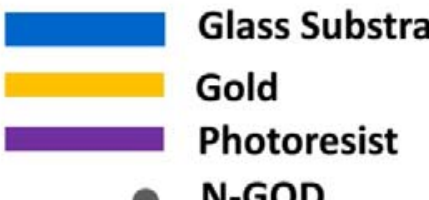

Photodetector

Figure 7. Schematic diagrams illustrating the fabrication process of the N-GQDs photodetector. 


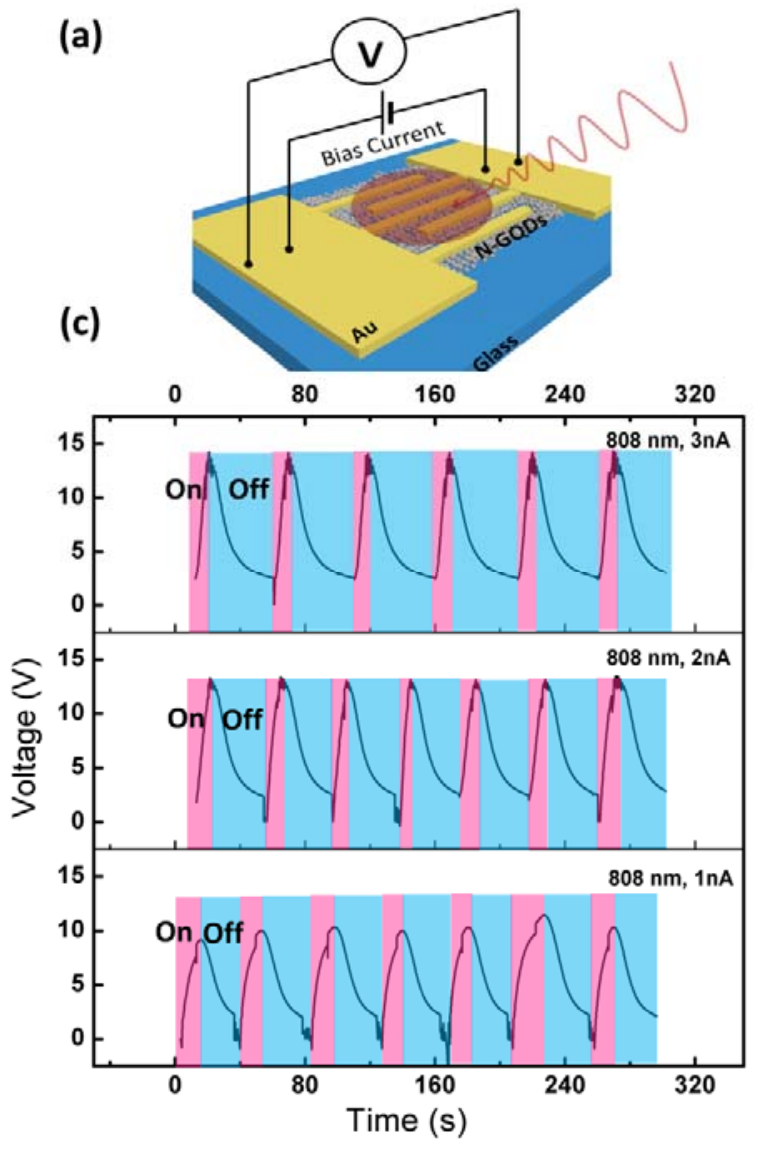

(b)

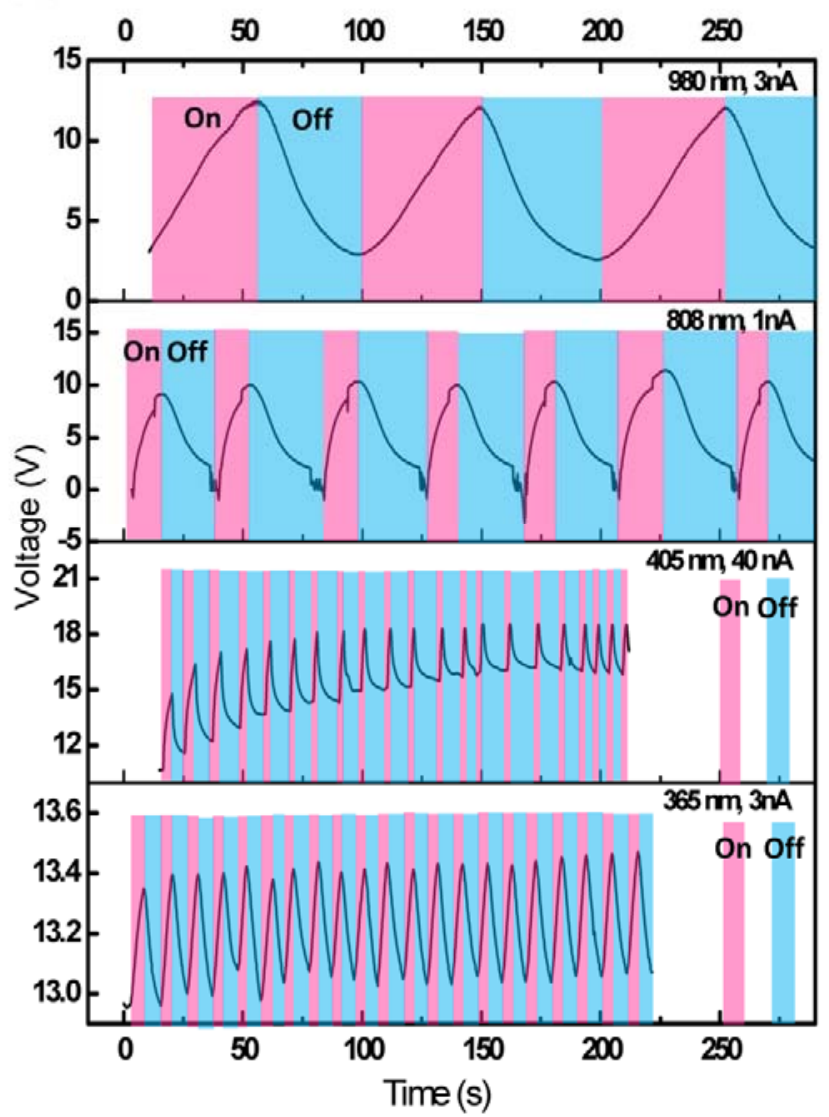

Figure 8. (a) The schematic diagram of the N-GQDs photodetector. (b) The photoresponse of the N-GQDs $(\Phi=4.0 \mathrm{~nm})$ with various light sources. (c) The photovoltage responses with various bias currents. 